\title{
JALONS POUR UNE HISTOIRE DES SCIENCES DE L'HOMME ÉTUDES DE CAS ET ESSAIS DE SYNTHÈSE
}

L'ensemble d'articles proposé ici au lecteur résulte d'un effort en vue de poser (en France, mais en s'inspirant d'exemples étrangers et de comparaisons internationales) les premiers jalons devant aider à la constitution d'une histoire des sciences de l'homme.

Cette publication est étroitement liée à la fondation (avril-juin 1986) de la Société française pour l'histoire des sciences de l'homme (SFHSH) dont la Revue de synthèse s'était déjà fait l'écho en son temps*. La Société, ayant par ses buts et par la pluralité d'appartenance de ses membres une vocation éminemment interdisciplinaire, trouve ainsi dans la Revue de synthèse, dont beaucoup d'objectifs sont proches des siens, une tribune pour ainsi dire amicale, et cela pour la seconde fois. Nous la remercions très vivement pour l'occasion qu'elle nous offre ainsi de présenter au lecteur quelques premiers travaux.

On ne manquera certes pas de relever bien des différences - voire des divergences - entre les textes ici présentés. Certains sont des bilans tantôt sereins, tantôt polémiques; d'autres sont des études de cas. Quelques-uns (distinction qui ne correspond pas à la précédente) sont principalement positifs, d'autres sont consciemment réflexifs, évaluatifs, voire critiques. Encore, parmi ces derniers, les jugements énoncés et les préférences exprimées quant à l'épistémologie, la méthodologie et l'insertion institutionnelle possibles d'une histoire des sciences de l'homme présentent-ils des divergences nombreuses et incontestables. Sans pratiquer un unanimisme de façade, il convient pourtant de souligner l'unicité du but poursuivi : développer en France (évidemment avec des vues comparatives) l'histoire des sciences de l'homme.

Certaines des divergences entre les articles tiennent tout naturellement

* Alain Drouard, Bernard-Pierre Lecuyer, Benjamin Matalon, " La situation de l'histoire des sciences de l'homme en France (Colloque de définition du C.N.R.S. et création d'une socièté scientifique) ", Revue de synthèse, IV S., 4, oct.-déc. 1986, p. 431-433.

Revue de synthèse: IV S. Nos 3-4, juil.-déc. 1988. 
(c'est un des postulats de base de la sociologie de la connaissance la plus classique) aux différences entre les situations épistémologiques, méthodologiques, institutionnelles, voire... existentielles (survie menacée ou non) dans lesquelles se trouvent leurs auteurs respectifs. Plus prosaïquement, de façon peut-être plus contingente mais en tout cas plus directe, il convient de préciser que les textes rassemblés ici ont deux origines sans doute connexes, mais néanmoins assez sensiblement distinctes.

Comme on l'indiquait dans le texte précité paru dans la Revue de synthèse en 1986, la création de la Société française pour l'histoire des sciences de l'homme est intervenue dans la foulée du Colloque de définition sur l'histoire des sciences sociales et humaines organisé par le Département des sciences de l'homme et de la société du C.N.R.S. les 14 et 15 avril 1986. Profitant de la mobilisation incontestable réalisée par ce colloque, la SFHSH fut immédiatement créée le soir mème du 15 avril 1986.

Ce rappel n'est pas pour l'anecdote. Certains des textes publiés ici sont directement issus du colloque. D'autres, au contraire, en sont indépendants, conçus spécifiquement ou adaptés spécialement pour ce numéro. Pourquoi souligner ainsi cette différence?

Le colloque "de définition" du C.N.R.S. se voulait essentiellement pragmatique : la " définition » dont il s'agissait était celle d'une politique visant à développer l'histoire des sciences de l'homme en France dans les cadres cognitifs et institutionnels existants. La base opératoire imposée d'emblée était celle des disciplines reconnues comme telles au C.N.R.S. Deux des articles publiés ci-après, celui de Charles C. Gillispie et celui de Stefan Collini, expriment la réaction de deux participants étrangers surpris par l'importance, excessive et erronée à leurs yeux, accordée aux disciplines actuelles et à leurs praticiens comme unités de base et comme acteurs majeurs dans cette histoire des sciences de l'homme encore à réaliser. Il y a peu à reprendre à leurs objections fortement argumentées, sinon le poids présent en France d'un cloisonnement de la vie scientifique dont il faut pour l'instant, faute de mieux, s'accommoder. Nous sommes en tout cas très reconnaissants à Stefan Collini d'avoir bien voulu réviser et adapter le texte de sa communication écrite au colloque pour le présenter ici, et à Charles Gillispie pour avoir rassemblé et rédigé ses impressions (hautement critiques, comme on le verra) sur ce même colloque.

Tous deux expriment la crainte que ce qu'ils appellent l' " histoire disciplinaire " ne traite que de problèmes issus des disciplines telles qu'elles existent aujourd'hui et donc que soient négliges des problèmes transversaux, tels que l'institutionnalisation ou la professionnalisation. 
Mais on peut se demander si de tels problèmes, bien qu'ils se posent dans toutes les disciplines, peuvent être abordés d'emblée dans leur généralité. Peut-on traiter de «la " professionnalisation en soi, ou n'eston pas contraint de parler de la professionnalisation de la psychologie, de l'histoire, de la linguistique? Certes, il faut être suffisamment attentif aux risques d'anachronisme, et garder présent à l'esprit que ces termes peuvent avoir désigné des contenus variables, et que d'autres mots peuvent avoir recouvert des pratiques qui ont évolué vers celles que nous connaissons. De telles précautions ne sont certainement pas hors de la portée des spécialistes d'une discipline, même s'il est vrai que certains tombent dans les pièges que Gillispie et Collini nous signalent ${ }^{1}$.

Ils redoutent aussi que l'histoire disciplinaire ne devienne une histoire apologétique racontant les inéluctables progrès vers l'état actuel de la science. Ce risque existe évidemment, et il n'est pas difficile de trouver des exemples de recherches historiques qui tombent sous le coup de cette critique. Mais on trouve aussi le risque inverse : on fait l'histoire de sa discipline parce qu'on est mécontent de son état actuel. C'est parfois une simple volonté institutionnelle, un compromis entre le désir d'abandonner un domaine de recherche auquel on ne croit plus et les exigences de la profession qui rendent difficile une reconversion à tout autre chose. Mais le mécontentement peut aussi orienter de façon plus spécifique vers l'histoire, ou éventuellement vers la sociologie ou l'épistémologie : il s'agit soit de montrer que le déroulement réel n'a pas été aussi harmonieux et rationnel qu'on le croit souvent, ou que des voies plus intéressantes ont été abandonnées, sous l'influence de facteurs externes. Certes, on peut estimer que cette histoire critique est aussi contestable que l'histoire apologétique, mais elle résulte plus que celle-ci d'une volonté de se dégager de la situation actuelle.

Outre les particularités de son objet (la connaissance et l'activité scientifiques), une des spécificités supplémentaires de l'histoire des sciences réside dans les motivations de ceux qui s'y intéressent, qu'ils soient auteurs ou simples lecteurs. On peut admettre que, dans d'autres domaines de l'histoire, on puisse chercher en premier lieu à savoir simplement "comment cela s'est réellement passé » (bien que, par exemple, la fonction de légitimation de l'histoire politique soit évidente). Pour ce qui est de l'histoire des sciences, on peut, plus qu'ailleurs, exclure cette curiosité pure : les préoccupations épistémologiques, ou

1. La SFSHS organise précisément un colloque sur l'institutionnalisation des sciences de l'homme en janvier 1989. 
éventuellement de politique de la recherche, sont probablement déterminantes pour orienter vers ce type d'intérêt, et tout particulièrement dans le cas des sciences de l'homme, encore incertaines quant à leurs méthodes, à leurs objets, et même quant à leur scientificité. On cherche dans le passé de quoi calmer, ou peut-être au contraire renforcer et justifier, ses inquiétudes. Il est significatif que, au C.N.R.S., l'histoire des sciences soit rattachée à la section de philosophie, et non à celle d'histoire, malgré quelques exceptions récentes. Sous-jacents à la plupart des recherches en histoire des sciences, on trouve donc des interêts qui amènent à viser des conclusions de portée générale, même quand l'impulsion de départ est donnée par une interrogation spécifique à une discipline. Les historiens ne cherchent plus guère les "lois de l'histoire", mais de nombreux historiens des sciences rêvent de mettre en évidence les lois du développement scientifique.

Une grande partie des réflexions qui précèdent s'appliquerait à toute histoire des sciences, sciences de la nature comprises. Y a-t-il une spécificité, de ce point de vue, des sciences de l'homme? L'existence, en France comme dans d'autres pays, de sociétés distinctes, par exemple, est-elle contingente, attribuable simplement au fait que l'histoire des sciences de l'homme est apparue alors que celle des sciences de la nature était déjà bien constituée, avec ses traditions et ses institutions? Ce qui apparait, et ce qui se trouve bien visible dans les textes qui suivent, c'est que l'objet des sciences de l'homme n'est pas déterminé de façon claire, qu'il a varié au cours du temps, que des approches acceptées à un certain moment ont été rejetées plus tard. A première vue, il n'en va pas de même, par exemple, en physique : au moins à partir de la fin du XVIr ${ }^{e}$ siècle, son objet semble déterminé de façon stable, et ceux qui s'en occupent appartiennent à une communauté bien structurée et institutionnalisée. Ce qui est à l'extérieur n'est pas de la physique, même si on peut trouver intéressant d'en faire l'histoire, comme l'astrologie, par exemple. On peut aborder de nombreux problèmes sans avoir à se demander longtemps s'ils touchent ou non à l'histoire de la discipline. Par contraste, presque tous les articles réunis ici (tout particulièrement celui de E. Apfelbaum et les contributions de J. Jamin et C. Blanckaert) se posent des problèmes quant à l'objet de leur discipline, et à ses variations au cours du temps. C'est même cette interrogation qui est souvent préconisée comme garde-fou contre les risques d'une histoire trop liée aux problèmes actuels. Pour se prémunir contre les risques d'une histoire téléologique, on s'efforce de montrer que l'objet ou les méthodes de la discipline auraient pu être autres, que ce sont des facteurs externes qui ont amené à l'état actuel.

Ce genre d'interrogation sur l'objet et les limites de leur discipline 
ne semble guère avoir préoccupé les historiens des sciences de la nature. On trouve bien, de temps en temps, des physiciens qui se demandent pourquoi on ne s'est jamais inquièté, par exemple, d'expliquer la formation de la mousse de la bière, mais ces interrogations sont restées isolées, et n'ont été à l'origine, à notre connaissance, ni de travaux d'historiens qui se seraient demandé comment ces problèmes ont été occultés, ni de recherches de physiciens visant à combler ces lacunes. Dans les sciences de l'homme, ces problèmes ont bien été abordés par les historiens, mais ne semblent pas plus avoir infléchi le déroulement des recherches.

C'est à dessein qu'on a insisté dans cette présentation sur les aspects problématiques et sur les débats plus que sur les résultats acquis. Remarquons simplement que les articles réunis ici, à peu près tous écrits par des spécialistes de leur discipline, sont loin d'être uniformément apologétiques ou téléologiques. On y relève aussi une démarche critique, ou plus simplement cette distance réfléchie envers l'objet qui est le propre des historiens. L'ambition commune des auteurs de ces textes est bien d'orienter fermement leurs travaux et ceux de leurs collègues vers la constitution en France d'une véritable histoire des sciences de l'homme.

Benjamin MataloN, Président de la SFHSH, Université Paris VIII.
Bernard-Pierre LÉCUYER, Secrétaire général de la SFHSH, GEMAS/CNRS. 